\title{
On the role of storm duration in the mapping of rainfall to flood return periods
}

\author{
A. Viglione and G. Blöschl \\ Institut für Wasserbau und Ingenieurhydrologie, Technische Universität Wien, Austria \\ Received: 7 October 2008 - Published in Hydrol. Earth Syst. Sci. Discuss.: 1 December 2008 \\ Revised: 28 January 2009 - Accepted: 12 February 2009 - Published: 19 February 2009
}

\begin{abstract}
While the correspondence of rainfall return period $T_{P}$ and flood return period $T_{Q}$ is at the heart of the design storm procedure, their relationship is still poorly understood. The purpose of this paper is to shed light on the controls on this relationship. To better understand the interplay of the controlling factors we assume a simplified world with block rainfall, constant runoff coefficient and linear catchment response. We use an analytical derived flood frequency approach in which, following design practise, $T_{P}$ is defined as the return period of the intensity-duration-frequency (IDF) curve given storm duration and depth. Results suggest that the main control on the mapping of rainfall to flood return periods is the ratio of storm duration and catchment response time, as would be expected. In the simple world assumed in this work, $T_{Q}$ is always smaller or equal than $T_{P}$ of the associated storm, i.e., $T_{Q} / T_{P} \leq 1$. This is because of the difference in the selectiveness of the rectangular filters used to construct the IDF curves and the unit hydrograph (UH) together with the fact that different rectangular filters are used when evaluating the storm return periods. The critical storm duration that maximises $T_{Q} / T_{P}$ is, in descending importance, a function of the catchment response time and the distribution of storm duration, while the maximum value of $T_{Q} / T_{P}$ is mainly a function of the coefficient of variation of storm duration. The study provides the basis for future analyses, where more complex cases will be examined.
\end{abstract}

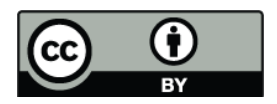

Correspondence to: A. Viglione (viglione@hydro.tuwien.ac.at)

\section{Introduction}

Flood design generally requires the estimation of flood discharges of a given return period at a site. If long stream flow records are available, the flood estimates can be derived directly from data by frequency analysis. If no or limited stream flow data are available, or floods associated with very large return periods are of interest, design floods are generally estimated based on design storms (e.g. Pilgrim and Cordery, 1993, p. 9.13). In this procedure, one or more storms of a given return period are used as an input to a rainfall-runoff model, and it is then assumed that the simulated peak discharge has the same return period (e.g. Packman and Kidd, 1980; Bradley and Potter, 1992). There have been warnings that this assumption may be grossly in error (e.g. Linsley et al., 1988, p. 365), and Pilgrim and Cordery (1975, p. 81) remarked: "The actual relationship between the frequencies of the design rainfall and the derived flood is obscure as each part of the overall design model introduces some joint probability". There are a number of "parts" that need to be considered which include storm rainfall intensity, storm duration, temporal and spatial storm patterns, and antecedent soil moisture conditions.

Despite the "obscure nature" of the mapping of return periods, there has been little work devoted to this issue. The main emphasis has been on finding the rainfall patterns or model parameters that give a close match between rainfall and flood return periods. Alfieri et al. (2008) assessed the accuracy of literature design hyetographs in producing flood peaks with the same return period as the storms. They noted that the estimation is biased in most of the cases and provided a correction factor to obtain more robust estimates of the design flood. Packman and Kidd (1980) investigated which antecedent conditions consistently gave flows that matched an observed flood frequency distribution. They found these

Published by Copernicus Publications on behalf of the European Geosciences Union. 
conditions to be related to average annual rainfall at several locations in the United Kingdom. For the simple case of the Probabilistic Rational Method, this problem has been directly addressed by estimating design runoff coefficients in a way that the design storms of a given return period produce flood peaks that match the observed flood peaks with the same return period (Pilgrim and Cordery, 1993, p. 9.20).

These studies are "black box" analyses where the emphasis is on identifying the optimum parameters in a design context. In contrast, the aim of this paper is to help understand why the return periods of rainfall and floods are different. Not only is this hoped to contribute to more informed flood design procedures, but this should also contribute to better assessing the magnitude of observed floods. For example, based on frequency analyses of runoff and rainfall data, Gutknecht et al. (2002) found that the 2002 Kamp flood in northern Austria had a flood return period of more than 1000 years while the return period of the associated rainfall was smaller. The difference of these magnitudes can be better understood if the controls on the relationship of the return periods are known.

As the emphasis in this paper is on understanding the controls, we have chosen to examine a highly simplified "world". We assume:

- block rainfall,

- constant runoff coefficient,

- linear catchment response.

This allows us to explicitly scrutinise the effect of storm duration which we consider the most basic control. The design storm procedure is mimicked in the domain of frequency distributions by an analytical derived distribution approach which facilitates the probabilistic interpretation. While the model is not complete in that it does not represent all possible processes, it is simple enough to actually understand the interplay of the controls. This paper is hence considered as a first step towards understanding more complex situations, where other processes are introduced such as the variability of the runoff coefficient, the complexity of the shapes of the hyetographs and multiple storms.

\section{Design-storm procedure and definition of storm re- turn period}

The idea of the design-storm procedure is to estimate a flood of a selected return period from rainfall intensity-durationfrequency (IDF) curves for the site of interest. In many cases, the hydrological engineer has standard IDF curves available for the site but it is important to understand the procedure used to develop them. For each duration selected, the annual maximum rainfall intensity is extracted from historical rainfall records. Then frequency analysis is applied to the annual data obtaining a return period for each intensity and duration.
What is termed "duration" in the procedure is in fact not a storm duration but an aggregation time interval, or aggregation level. For example, if hourly rainfall data are available and one is interested in the IDF relationship for a duration of $3 \mathrm{~h}$, one runs a moving averaging window over the hourly data and extracts the largest 3-h average of each year to do the frequency analysis. The moving averaging procedure is equivalent to convoluting the rainfall time series with a rectangular filter (with a base of $3 \mathrm{~h}$ in the example).

The way the design storm method is applied varies considerably between countries (Pilgrim and Cordery, 1993, p. 9.13) but the main components of the procedure can be summarised as following:

1. Selection of many storms of different durations reading off their mean intensities from the IDF curve corresponding to the return period $T_{P}$ of interest. As noted above, rainfalls from the IDF curves do not represent complete storms but are from intense bursts within these storms. The storm duration may hence differ from the aggregation level used to read off the intensity from the IDF curve. However, in many cases storm duration is chosen equal to the aggregation level (see Chow et al., 1988, for details).

2. Application of rainfall time patterns to these storms (design hyetograph). Rigorously, the design temporal patterns need to be appropriate for the intense bursts within storms, and not for complete storms (Pilgrim and Cordery, 1993, p. 9.13) but, again, in practise these two are often set equal.

3. Application of spatial patterns to rainfall or, more simply, of an areal reduction factor for catchment area.

4. Transformation of the design storm to a flood hydrograph using a runoff model calibrated for the catchment of interest.

5. Selection of the maximum flood peak of the flood hydrographs produced by storms of different durations.

It is then assumed that this flood peak has a return period $T_{Q}$ equal to $T_{P}$.

In the real world there is no rigorous solution to the problem of choosing the design parameters (i.e., the shape of the hyetograph, the rainfall-runoff model parameters, etc.) in a way that $T_{Q}$ matches $T_{P}$ because of the large number of controls that are difficult to understand.

In contrast, when a simplified world is assumed, the exact mapping of rainfall to flood return periods can be derived. In the case of block rainfall, as assumed here, the total rainfall event and the main burst are indeed identical, so the aggregation level used to evaluate the return period of a storm is equal to the duration of that storm. 


\section{The derived distribution approach in the simplified world}

We use here a derived distribution approach which consists of combining a statistical rainfall model with a deterministic rainfall-runoff model. This model represents the simplified world which we are meaning to analyse. The rainfall model assumes events to be uniform and independent, and durations and intensities to be random and mutually dependent. Other factors such as multiple storms, within-storm intensity patterns, seasonality and spatial variability of the rainfall intensities are deliberately neglected.

The rainfall-runoff model is event based. It assumes a constant runoff coefficient and linear routing represented by an exponential unit hydrograph. Any non-linearities in the system as well as random controls on runoff generation are neglected. The model mimics the design storm procedure which, in this simplified world, becomes straightforward: rectangular storms, whose intensities on the IDF curve correspond to their durations, are convoluted with the linear rainfall-runoff model from which the return period of the associated flood peaks can be estimated. In this paper, we have chosen to do most of the analyses in the probability domain rather than generating time series as the former is more efficient and directly provides insight into the controls.

\subsection{Derived flood return period}

Given the joint probability density function of rainfall intensity $i$ and storm duration $t_{r}$ as $f_{I, T_{r}}\left(i, t_{r}\right)$, the probability that $Y$, i.e., the peak discharge of all independent floods, does not exceed the flood peak value $q_{p}$ is

$$
F_{Y}\left(q_{p}\right)=\operatorname{Pr}\left[Y \leq q_{p}\right]=\iint_{R} f_{I, T_{r}}\left(i, t_{r}\right) d i d t_{r}
$$

where $R$ is the region of the $\left(i, t_{r}\right)$ space for which the combination of these two values is transformed into a peak smaller than or equal to $q_{p}$ by the rainfall-runoff model (see Figs. 1 and 2 in Wood, 1976, for a graphical representation).

This equation can be simplified if some assumptions are formulated. We use here a simplified version of the model of Sivapalan et al. (2005). For the rainfall model we assume that the storms are independent, the number of storm events per year is Poisson distributed (see Kottegoda and Rosso, 1997, p. 455) with mean $m$ (set to 40), and that the distribution $f_{T_{r}}\left(t_{r}\right)$ of the storm duration $t_{r}$ is known.

Depending on the case analysed in this paper, we assume a discrete distribution (Sect. 4.1) or assume that $t_{r}$ is continuously distributed according to a Weibull distribution (Sects. 4.2-4.4) with the probability density function (pdf):

$f_{T_{r}}\left(t_{r}\right)=\frac{\beta_{r}}{\gamma_{r}}\left(\frac{t_{r}}{\gamma_{r}}\right)^{\beta_{r}-1} \exp \left(-\frac{t_{r}}{\gamma_{r}}\right)^{\beta_{r}}$ where $\gamma_{r}$ and $\beta_{r}$ are the scale and shape parameters, respectively. The scale parameter is related to the mean storm duration $\delta_{r}$ by

$\gamma_{r}=\delta_{r}\left[\Gamma\left(1+\frac{1}{\beta_{r}}\right)\right]^{-1}$.

The shape parameter is related to the coefficient of variation of the distribution by

$C V_{r}=\sqrt{\frac{\Gamma\left(1+2 / \beta_{r}\right)}{\left[\Gamma\left(1+1 / \beta_{r}\right)\right]^{2}}-1}$.

We examine here a basic case of $\beta_{r}=0.7$ and $\delta_{r}=12 \mathrm{~h}$, and vary these parameters in some of the variants, as detailed in the results section.

The storm rainfall intensity $i$ is assumed to be distributed according to a gamma distribution (commonly used for areally averaged intensities, see e.g. Sivapalan and Blöschl, 1998):

$f_{I \mid T_{r}}\left(i \mid t_{r}\right)=\frac{\lambda}{\Gamma(\kappa)}(\lambda i)^{\kappa-1} \exp (-\lambda i)$,

where parameters $\lambda$ and $\kappa$ are functions of $t_{r}$.

Following Sivapalan et al. (2005) we assume that expectation and coefficient of variation of the intensities are related to the storm duration by power laws

$E\left[i \mid t_{r}\right]=a_{1} t_{r}^{b_{1}}$ and $C V^{2}\left[i \mid t_{r}\right]=a_{2} t_{r}^{b_{2}}$,

so that

$\kappa=\frac{t_{r}^{-b_{2}}}{a_{2}}$ and $\lambda=\frac{t_{r}^{-b_{1}-b_{2}}}{a_{1} a_{2}}$.

In all the presented analyses, we have assumed $a_{1}=1.05 \mathrm{~mm} \mathrm{~h}^{-b_{1}-1}, \quad b_{1}=0.01, \quad a_{2}=1.5$ and $b_{2}=-0.55$ as in Sivapalan et al. (2005) (values calibrated using data of the Frankenfels raingauge, northern Austria).

As the runoff model, we use a linear reservoir with constant response time $t_{c}$ and a runoff coefficient of unity. The transformation of rainfall to runoff can then be expressed by the convolution integral of the exponential unit hydrograph (UH) with rectangular storms, from which one can evaluate the flood peak:

$q_{p}=\Pi_{Q}\left(i, t_{r}\right)=i \cdot\left[1-\exp \left(-\frac{t_{r}}{t_{c}}\right)\right]$,

where $\Pi_{Q}$ is the runoff-model operator.

Adopting these simplifications, the integral of Equation (1) simplifies to

$F_{Y}\left(q_{p}\right)=\int_{0}^{\infty} F_{I \mid T_{r}}\left(\Pi_{Q}^{-1}\left(q_{p}, t_{r}\right) \mid t_{r}\right) f_{T_{r}}\left(t_{r}\right) d t_{r}$,

where $\Pi_{Q}^{-1}\left(q_{p}, t_{r}\right)=q_{p} /\left[1-\exp \left(-t_{r} / t_{c}\right)\right]$ is the storm intensity that, for given $t_{r}$ and $t_{c}$, produces the flood peak $q_{p}$, and 
$F_{I \mid T_{r}}\left(. \mid t_{r}\right)$ is the cumulative distribution of rainfall intensities conditioned on $t_{r}$.

The transition from the distribution of all floods $F_{Y}\left(q_{p}\right)$ to the distribution of the annual maximum floods $F_{Q}\left(q_{p}\right)$ is obtained by using the theory of order statistics (e.g. Kottegoda and Rosso, 1997, p. 455):

$$
F_{Q}\left(q_{p}\right)=\exp \left\{-m\left[1-F_{Y}\left(q_{p}\right)\right]\right\},
$$

which can also be expressed in terms of the return period (in years):

$T_{Q}=\left\{1-F_{Q}\left(q_{p}\right)\right\}^{-1}$.

The integral in Eq. (9) is evaluated numerically in the probability space.

\subsection{Derived IDF curves and storm return periods}

We exploit the similarity between the runoff modelling (convolution of rainfall by a UH) and the IDF procedure (convolution of rainfall by a moving averaging window) to derive the return period of rainfall for different aggregation levels from the statistical rainfall model. The difference between the runoff model and the IDF procedure is that the former uses an exponential filter while the latter uses rectangular filters, one for each aggregation level. The exponential filter represents, to some degree, hydrological processes in that it is a summary description of the non-linearity of surface and near surface runoff (velocity depending on depth), so the rising limb is always steeper than the falling limb of the hydrograph. In contrast, the rectangular filter that is used to construct the IDF curve is commonly chosen for convenience and tradition.

If we let a random variable $I$ denote the rainfall intensity of storms averaged on the aggregation level $t_{\mathrm{IDF}}$, the probability that this intensity is lower or equal to an intensity $\phi$ is termed $F_{I}\left(\phi, t_{\mathrm{IDF}}\right)$. To derive the cumulative distribution of $I$ (defined for a single $t_{\mathrm{IDF}}$ ), we proceed as in Eq. (1):

$F_{I}\left(\phi, t_{\mathrm{IDF}}\right)=\operatorname{Pr}[I \leq \phi]=\iint_{R^{\prime}} f_{I, T_{r}}\left(i, t_{r}\right) d i d t_{r}$,

where $R^{\prime}$ is the region of the $\left(i, t_{r}\right)$ space such that the combination of these two values is transformed to a value smaller or equal to $\phi$ by the IDF filter with aggregation level $t_{\text {IDFF }}$. The result of the rectangular filtering can be written as:

$\phi=\Pi_{P}\left(i, t_{r}\right)=\left\{\begin{array}{ll}i & \text { if } t_{\mathrm{IDF}} \leq t_{r} \\ i \cdot t_{r} / t_{\mathrm{IDF}} & \text { if } t_{\mathrm{IDF}}>t_{r}\end{array}\right.$.

With analogous simplifications as in Eq. (9), the cumulative distribution of $I$ is

$F_{I}\left(\phi, t_{\mathrm{IDF}}\right)=\int_{0}^{\infty} F_{I \mid T_{r}}\left(\Pi_{P}^{-1}\left(\phi, t_{r}\right) \mid t_{r}\right) f_{T_{r}}\left(t_{r}\right) d t_{r}$,

where $\Pi_{P}^{-1}\left(\phi, t_{r}\right)$ is the inverse function of Eq. (13) and expresses the intensity of a storm of duration $t_{r}$ that has average

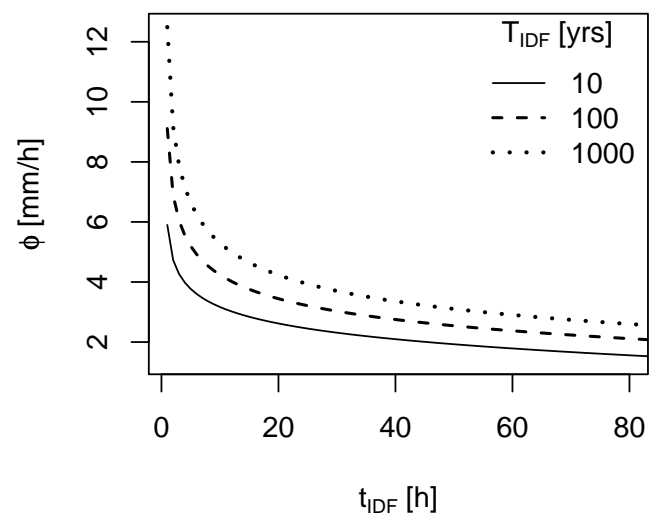

Fig. 1. Example IDF curves with parameters $a_{1}=1.05, b_{1}=0.01$, $a_{2}=1.5, b_{2}=-0.55, \beta_{r}=0.7$ and $\delta_{r}=12 \mathrm{~h}$ of the rainfall model. $\phi$ is the rainfall intensity over the aggregation level $t_{\mathrm{IDF}}$ and $T_{\mathrm{IDF}}$ is the IDF return period.

intensity $\phi$ over the aggregation level $t_{\mathrm{IDF}}$. The transition from the distribution $F_{I}\left(\phi, t_{\mathrm{IDF}}\right)$ of all intensities over one aggregation level $t_{\mathrm{IDF}}$ to the distribution $F_{P}\left(\phi, t_{\mathrm{IDF}}\right)$ of the annual maximum rainfall intensities $P$ yields

$F_{P}\left(\phi, t_{\mathrm{IDF}}\right)=\exp \left\{-m\left[1-F_{I}\left(\phi, t_{\mathrm{IDF}}\right)\right]\right\}$,

which can also be expressed in terms of the return period (in years) as

$T_{\mathrm{IDF}}\left(\phi, t_{\mathrm{IDF}}\right)=\left\{1-F_{P}\left(\phi, t_{\mathrm{IDF}}\right)\right\}^{-1}$.

This equation represents the IDF curves, an example of which is provided in Fig. 1.

In our simplified world, the return period of individual storms can now be read off the IDF curve as $T_{\mathrm{IDF}}\left(\phi=i, t_{\mathrm{IDF}}=t_{r}\right)$. The return period $T_{P}$ of the storms that produce the maximum annual peaks $q_{p}$ (here called floodproducing storms) is then

$T_{P}=T_{\mathrm{IDF}}\left(\phi=\Pi_{Q}^{-1}\left(q_{p}, t_{r}=t_{\mathrm{IDF}}\right), t_{\mathrm{IDF}}=t_{r}\right)$

where $\Pi_{Q}^{-1}($.$) is the storm intensity that, for given t_{r}$ and $t_{c}$, produces the flood peak $q_{p}$.

\section{Results}

The mapping of rainfall to flood return periods is now shown by graphs that relate the return periods $T_{Q}$ and $T_{P}$ of the same event. Different storm durations are drawn as different lines in the $\left(T_{P}, T_{Q}\right)$ space. The envelope of these lines, which maximises the ratio $T_{Q} / T_{P}$, is the result of the design storm procedure described in Sect. 2. We term critical storm duration(s), $t_{r}^{*}$, the duration(s) for which $T_{Q} / T_{P}$ is maximised. 

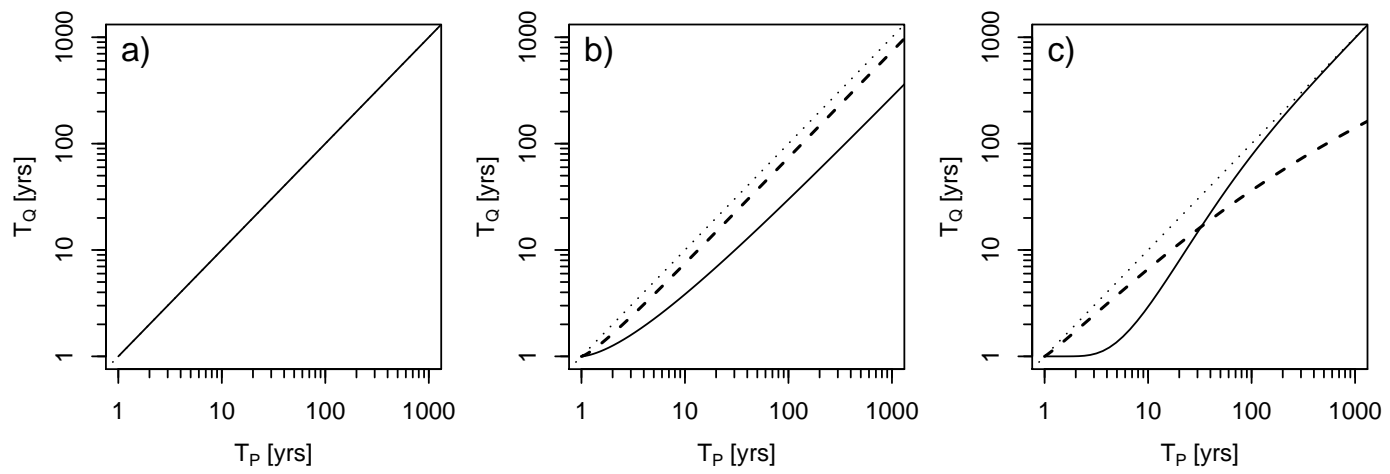

Fig. 2. Relation between rainfall return periods $T_{P}$ and flood return periods $T_{Q}$ for discrete distributions of storm durations: (a) only one possible storm duration $t_{1}$; (b) only two possible storm durations $t_{1}=6 \mathrm{~h}$ (continuous line) and $t_{2}=12 \mathrm{~h}$ (dashed line); (c) only two possible storm durations $t_{1}=3 \mathrm{~h}$ (continuous line) and $t_{2}=144 \mathrm{~h}$ (dashed line). The response time of the catchment is always $t_{c}=6 \mathrm{~h}$.

\subsection{Simple case: discrete number of storm durations}

First, we consider some extremely simplified cases. Suppose to have only one possible storm duration $t_{1}$, so that its probability is $p_{T_{r}}\left(t_{1}\right)=1$. In this limiting case, as storms differ by their intensity only, $q_{p}$ is a strictly monotonically increasing function of $i$ and a perfect correspondence $T_{P}=T_{Q}$ follows (Fig. 2a).

If, instead, two storm durations $t_{1}$ and $t_{2}$ are possible with equal probabilities $p_{T_{r}}\left(t_{1}\right)=p_{T_{r}}\left(t_{2}\right)=1 / 2$, this is no longer true. In Fig. $2 \mathrm{~b}$ we have imposed $t_{1}=t_{c}$ and $t_{2}=2 t_{c}$. The graph suggests that $T_{Q}$ is always lower than $T_{P}$. This result can be explained by the differences between the distribution of the flood peak $q_{p}$ and the distributions of the intensities that can cause it, i.e., $\Pi_{Q}^{-1}\left(q_{p}, t_{1}\right)$ and $\Pi_{Q}^{-1}\left(q_{p}, t_{2}\right)$. From Equation (9) it follows that

$$
\begin{aligned}
F_{Y}\left(q_{p}\right)= & \frac{1}{2}\left[F_{I \mid T_{r}}\left(\Pi_{Q}^{-1}\left(q_{p}, t_{1}\right) \mid t_{1}\right)+\right. \\
& \left.+F_{I \mid T_{r}}\left(\Pi_{Q}^{-1}\left(q_{p}, t_{2}\right) \mid t_{2}\right)\right] .
\end{aligned}
$$

On the other hand, since two durations are possible, two distributions $F_{I}$ have to be considered. For the storms of duration $t_{1}$ (continuous line in Fig. 2b), given that

$$
\Pi_{P}^{-1}\left(\Pi_{Q}^{-1}\left(q_{p}, t_{1}\right), t_{1}\right)=\Pi_{P}^{-1}\left(\Pi_{Q}^{-1}\left(q_{p}, t_{1}\right), t_{2}\right)=\Pi_{Q}^{-1}\left(q_{p}, t_{1}\right),
$$

Equation (14) becomes

$$
\begin{aligned}
F_{I}\left(\Pi_{Q}^{-1}\left(q_{p}, t_{1}\right), t_{1}\right)= & \frac{1}{2}\left[F_{I \mid T_{r}}\left(\Pi_{Q}^{-1}\left(q_{p}, t_{1}\right) \mid t_{1}\right)+\right. \\
& \left.+F_{I \mid T_{r}}\left(\Pi_{Q}^{-1}\left(q_{p}, t_{1}\right) \mid t_{2}\right)\right] .
\end{aligned}
$$

The only difference between $F_{Y}$ and $F_{I}$ is in the second part of the second term of Eqs. (18) and (20). As $t_{1}<t_{2}$, it follows that $F_{I \mid T_{r}}\left(\Pi_{Q}^{-1}\left(q_{p}, t_{1}\right) \mid t_{2}\right)$ is greater than $F_{I \mid T_{r}}\left(\Pi_{Q}^{-1}\left(q_{p}, t_{2}\right) \mid t_{2}\right)$ and, as a consequence, that $F_{I}\left(\Pi_{Q}^{-1}\left(q_{p}, t_{1}\right), t_{1}\right)>F_{Y}\left(q_{p}\right)$. Since they transform to the extremes in the same way, $T_{P}>T_{Q}$.
Considering the storms of duration $t_{2}$ (dashed line in Fig. 2b) Eq. (14) becomes

$$
\begin{aligned}
F_{I}\left(\Pi_{Q}^{-1}\left(q_{p}, t_{2}\right), t_{2}\right)=\frac{1}{2}[ & F_{I \mid T_{r}}\left(\Pi_{Q}^{-1}\left(q_{p}, t_{2}\right) \cdot \frac{t_{2}}{t_{1}} \mid t_{1}\right)+ \\
& \left.+F_{I \mid T_{r}}\left(\Pi_{Q}^{-1}\left(q_{p}, t_{2}\right) \mid t_{2}\right)\right] .
\end{aligned}
$$

Here the difference between $F_{Y}$ and $F_{I}$ is in the first part of the second term of Eqs. (18) and (21). As $t_{1}<t_{2}$, it follows that $F_{I}\left(\Pi_{Q}^{-1}\left(q_{p}, t_{2}\right), t_{2}\right)>F_{Y}\left(q_{p}\right)$, so $T_{P}>T_{Q}$.

Heuristically, the finding of $T_{Q}$ always being lower than $T_{P}$ has to do with (a) the shape of the UH being different from the shape of the IDF filter and (b) the fact that two different filters are used for assigning $T_{P}$ while a single filter is used for assigning $T_{Q}$. The rectangular IDF filter of aggregation level $t_{1}$ strongly highlights the events of duration $t_{1}$, more than the exponential UH does. In the same way, the IDF filter of aggregation level $t_{2}$ strongly highlights the events of duration $t_{2}$. The $\mathrm{UH}$ is less selective and easily allows events of both durations to produce maximum peaks.

Another system is shown in Fig. $2 c$ that differs from $2 b$ in that $t_{1} \ll t_{c}$ and $t_{2} \gg t_{c}$. In this case, for high return periods $T_{Q}$ is similar to $T_{P}$ of short storms (duration $t_{1}$ ), for low return periods $T_{Q}$ is similar to $T_{P}$ of long storms (duration $t_{2}$ ), while in the middle part $T_{Q}$ is lower than $T_{P}$ for both types of storms. This behaviour is a consequence of the shape of $f_{I \mid T_{r}}\left(i \mid t_{r}\right)$, i.e., the distribution of intensities given the storm durations. Consider once more the case $t_{\mathrm{IDF}}=t_{1}$ (continuous line in Fig. 2c) for which Eqs. (18) and (20) hold. For high values of $q_{p}$, the terms $F_{I \mid T_{r}}\left(\Pi_{Q}^{-1}\left(q_{p}, t_{1}\right) \mid t_{2}\right)$ and $F_{I \mid T_{r}}\left(\Pi_{Q}^{-1}\left(q_{p}, t_{2}\right) \mid t_{2}\right)$, that are the ones that cause the differences between $T_{P}$ and $T_{Q}$, tend to be equal to 1 . Therefore $T_{Q} \approx T_{P}$. Analogous considerations can be used to explain the behaviour of the dashed line corresponding to $t_{\mathrm{IDF}}=t_{2}$. For low values of $q_{p}$, the terms $F_{I \mid T_{r}}\left(\Pi_{Q}^{-1}\left(q_{p}, t_{1}\right) \mid t_{1}\right)$ and $F_{I \mid T_{r}}\left(\Pi_{Q}^{-1}\left(q_{p}, t_{2}\right) \cdot \frac{t_{2}}{t_{1}} \mid t_{1}\right)$ tend both to 0.

Hydrologically, the most extreme short duration storms result in relatively large floods (larger than those from the most 

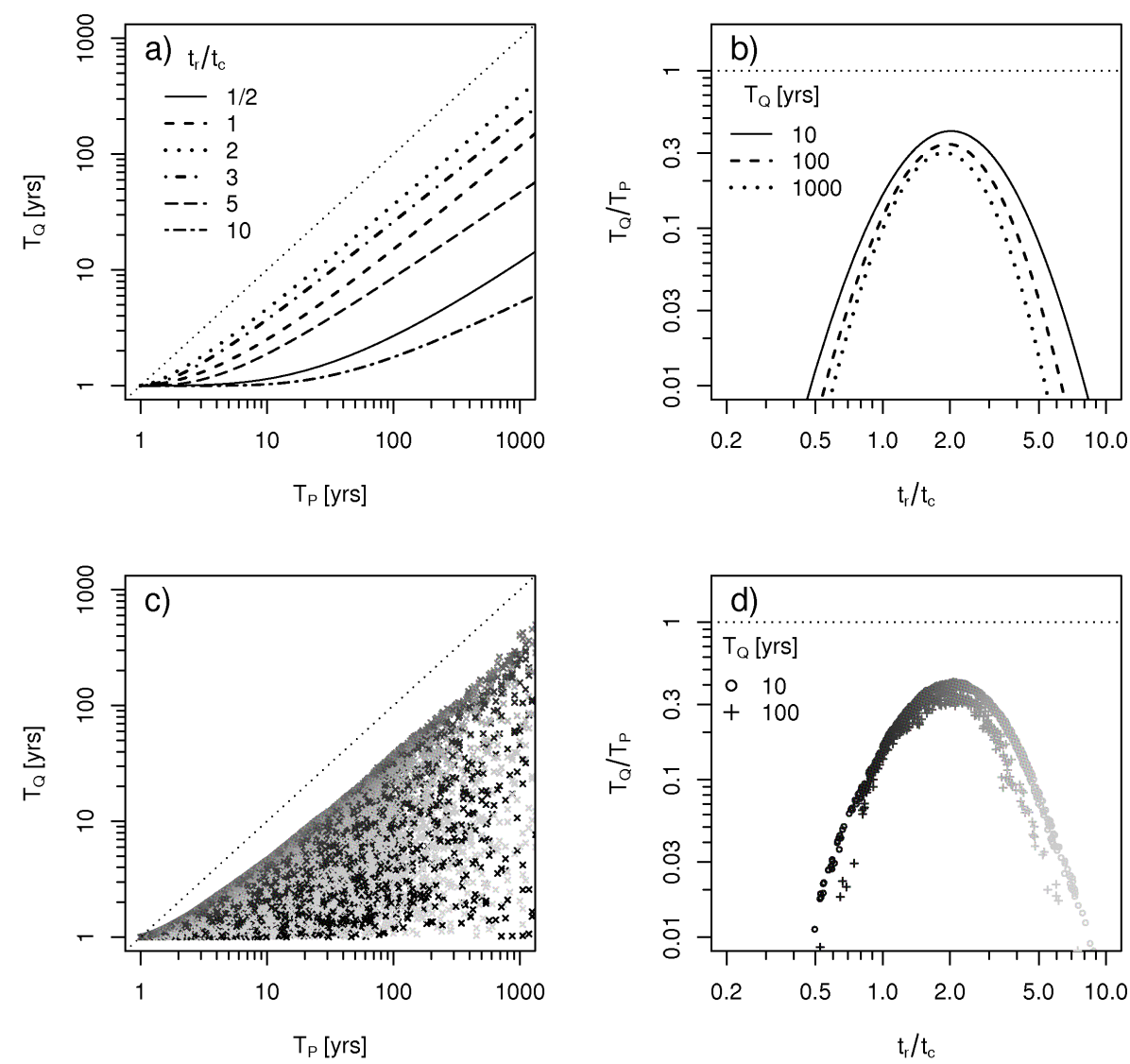

Fig. 3. Relation between rainfall return periods $T_{P}$ and flood return periods $T_{Q}$ for different storm durations $t_{r}$. Case of response time $t_{c}$ equal to the mean storm duration $\delta_{r}\left(t_{c}=\delta_{r}=12 \mathrm{~h}\right)$. The two upper graphs $(\mathbf{a}, \mathbf{b})$ are obtained with the analytical derivation of $T_{P}$ and $T_{Q}$; the two lower graphs $(\mathbf{c}, \mathbf{d})$ are the result of a Monte-Carlo simulation (20000 simulated years). The grey-scale of points represents storm durations (dark points are short storms). For the slices in graph (d), flood return periods between 8 and 13 years have been labelled as 10 yrs and those between 50 and 200 years have been labelled as $100 \mathrm{yrs}$.

extreme long duration storms) because of the shape of the IDF curve. The smallest long duration storms result in relatively large floods because saturation of the catchment is reached.

In the case of Fig. 2a the design storm procedure will give a 1:1 mapping of the return periods. In the case of Fig. $2 b$, $t_{2}$ will be the critical storm duration $\left(t_{r}^{*}=t_{2}\right)$. It is interesting to see that in Fig. 2c the highest maximum annual floods are given by rainfall events with different durations, depending on the storm return period considered, so $t_{r}^{*}$ is not unique for the catchment.

Analogous results can be obtained when 3 or more storm durations are possible. The generalised case is given by the continuous distribution of storm durations shown in the next section.

\subsection{Continuous distribution of storm durations}

Storm duration $t_{r}$ is now considered to be distributed according to the Weibull distribution of Eq. (2). Figure 3 shows the relationship between $T_{P}$ and $T_{Q}$ for different storm durations $t_{r}$ when the response time of the linear reservoir $t_{c}$ is equal to the average annual storm duration $\delta_{r}$. The mapping of the return periods is plotted in the upper-left graph. The six curves represent 6 storm durations as multiples of $t_{c}$.

The envelope curve in Fig. 3a is always below the 1 to 1 line indicating that $T_{Q}$ obtained by the design storm procedure is smaller than $T_{P}$. This can be explained analytically by generalizing the comparison between Eqs. (18), (20) and (21) to the continuous case, and analogous hydrological arguments apply as in the discrete case. $T_{P}$ is greater than $T_{Q}$ because the IDF filters are more selective than the UH filter. This is because of their shapes (rectangular as opposed to exponential) and the fact that, for each storm duration, a different filter is used when constructing the IDF curve while the same UH is used for all storms.

This behaviour is further illustrated by slicing Fig. 3a horizontally, and expressing the slices in terms of the ratio of return periods $T_{Q} / T_{P}$. This ratio is shown in Fig. $3 \mathrm{~b}$ as a function of storm duration $t_{r}$ for three flood return periods $T_{Q}$ (10, 100 and 1000 years). These curves are obtained by 

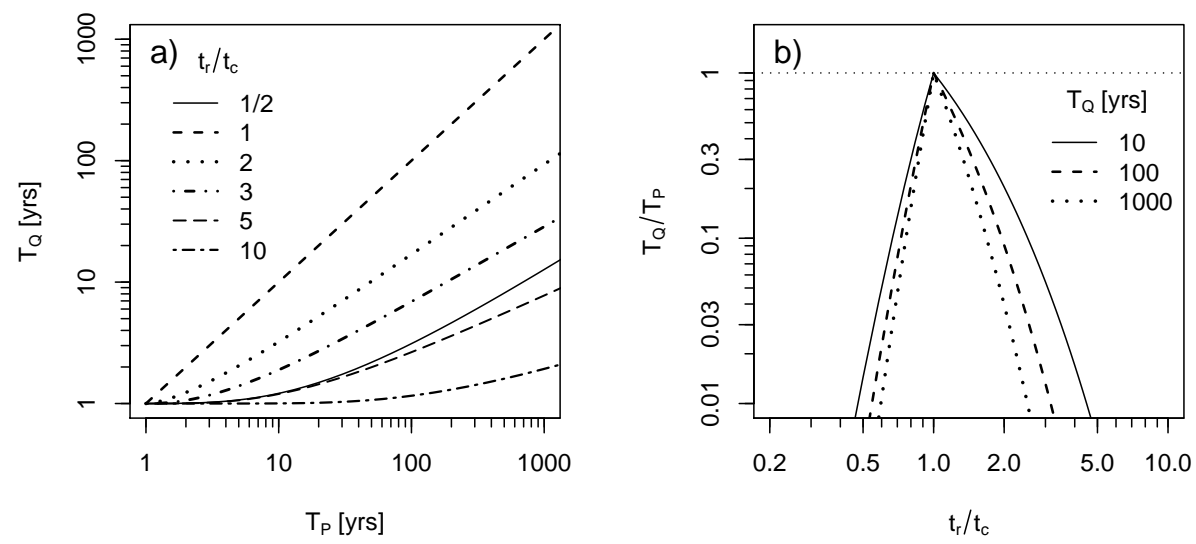

Fig. 4. Relation between rainfall return periods $T_{P}$ and flood return periods $T_{Q}$ for different storm durations $t_{r}$. Case of rectangular UH with width equal to $t_{c}\left(t_{c}=\delta_{r}=12 \mathrm{~h}\right)$.

interpolations in the $\left(T_{P}, T_{Q}, t_{r}\right)$ space. For short storm durations, the ratio of the return periods increases with storm duration, reaches a maximum around $t_{r}^{*} \approx 2 t_{c}$ (the critical storm duration), and decreases for longer durations. At the maximum, the difference between $T_{P}$ and $T_{Q}$ slightly depends on the considered value of $T_{Q}$, and $T_{Q} / T_{P}$ is always below 0.5 .

It appears that, for short durations, peak flows are small and hence the ratio $T_{Q} / T_{P}$ is small. During short duration storms, the catchment does not contribute entirely to runoff generation, so the storms must be extremely severe to produce big floods. The ratio at short durations is therefore controlled by runoff response. For long durations, peak flows are also small and hence the ratio $T_{Q} / T_{P}$ is small. This is because, in this case, runoff has already reached equilibrium (the catchment totally contributes to runoff) but, according to the IDF curves, rainfall intensities are very low. The ratio at long durations is therefore controlled by rainfall. The maximum of the curves in Fig. $3 \mathrm{~b}$ is due to the interplay between catchment processes and rainfall processes and occurs at the critical storm duration $t_{r}^{*}$.

To be able to plot individual events, Monte-Carlo simulations have been performed based on the same assumptions for the rainfall and rainfall-runoff models. The results are hence fully consistent with the analytical approach. A scatter plot of $T_{P}$ versus $T_{Q}$ based on the Monte-Carlo simulations is shown in Fig. 3c. Each point represents one maximum annual flood and its corresponding storm. The scatter comes from random storm durations. The grey scale used in the graph is related to the duration of the storms: dark points are short storms, light-grey points are long storms. Two slices through Fig. $3 \mathrm{c}$ represented in terms of the ratio $T_{Q} / T_{P}$ are shown in Fig. 3d. The points, each one representing one event, clearly trace the analytical curves of Fig. 3b. As can be seen in the graphs, the simulated events are more dense around the critical storm duration (corresponding to the envelope curve in Fig. 3c), meaning that the catchment acts as a filter on storm durations. This is an important effect that cannot be obtained by the analytical approach, and is discussed in more detail in Sect. 4.4.

In Sect. 4.1 we have stressed that the shape of the $\mathrm{UH}$ is an important control on the relation $\max \left(T_{Q} / T_{P}\right)<1$. We hence expect the behaviour of the mapping to change when changing the shapes of the UH. In the limiting case, where the UH is a rectangular function of width $t_{c}$, the resulting mapping is shown in Fig. 4. In this case, the critical storm duration is $t_{r}^{*}=t_{c}$ and gives $T_{P}=T_{Q}$. This is not surprising, because the convolution of the rainfall series with the rectangular $\mathrm{UH}$ is identical to the IDF filtering. The series of maximum peaks $q_{p}$ and the series of the peak intensities $\phi$ (with $t_{\mathrm{IDF}}=t_{c}$ ) coincide. For shorter and longer storms than $t_{c}$, the flood return periods are of course smaller than the rainfall return period.

For the case of a rectangular UH, the design storm method would hence be exact yielding the flood return period equal to the rainfall return period. UH shapes that lie between exponential and rectangular have been represented by a beta distribution, as in Alfieri et al. (2008). Results (not shown here) indicate that the envelope curves lie between those represented in Figs. 3a and 4a.

\subsection{Effect of average storm duration, catchment response time and return period}

After these first examples, we investigate the combined effect on the $\left(T_{P}, T_{Q}\right)$ mapping of the average storm duration $\delta_{r}$, the catchment response time $t_{c}$ and the range of return periods $T$ considered. Figure 5 is analogous to Fig. 3ab except for $t_{c}>\delta_{r}\left(t_{c}=60 \mathrm{~h}, \delta_{r}=12 \mathrm{~h}\right)$. In this case, the critical storm duration visibly depends on the return period which was not the case for $t_{c}=\delta_{r}=12 \mathrm{~h}$ (Fig. 3). For low return periods, $t_{r}^{*} \approx t_{c}$, while for high return periods $t_{r}^{*} \approx 2 t_{c}$.

This is the effect of the interplay of the catchment response time $t_{c}$ and the distribution of storm durations. Almost all storms are short (relative to $t_{c}$ ), so regular floods are produced by short storms. Only very extreme floods are 

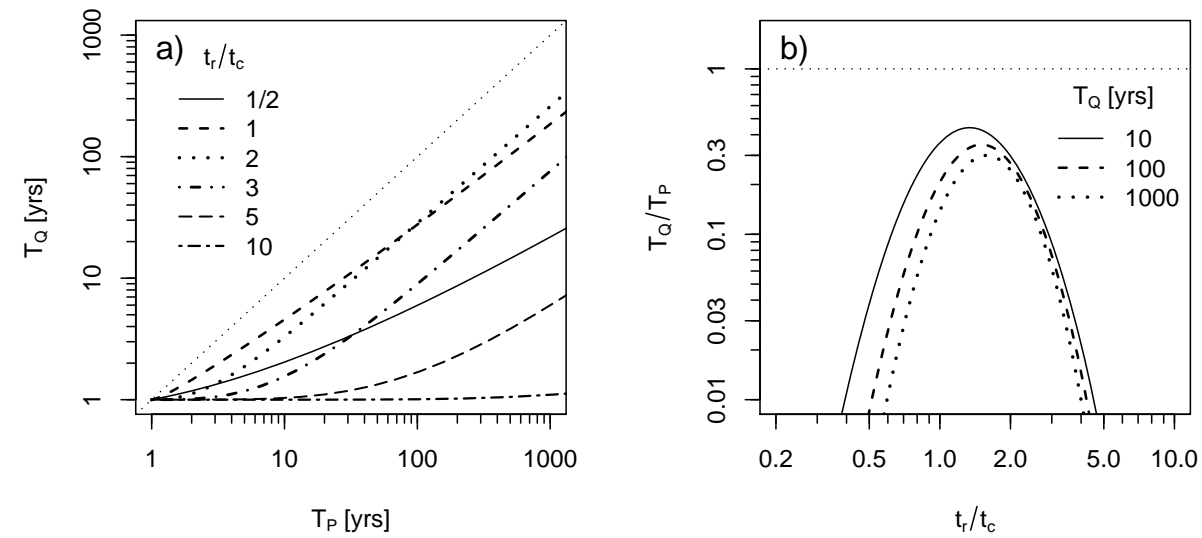

Fig. 5. Relation between rainfall return periods $T_{P}$ and flood return periods $T_{Q}$ for different storm durations $t_{r}$. Case of exponential UH with response time $t_{c}$ greater than the mean storm duration $\delta_{r}\left(t_{c}=60 \mathrm{~h}, \delta_{r}=12 \mathrm{~h}\right)$.

produced by (rare) longer duration storms resulting in larger $t_{r}^{*}$ as the return period increases. Interestingly, for the converse case $\left(t_{c}=60, \delta_{r}=12 \mathrm{~h}\right) t_{r}^{*}$ decreases from about $3 t_{c}$ to about $2 t_{c}$ as the return period increases (not shown here). Apparently, for the limit of very large return periods, the limiting critical storm duration $t_{r}^{*}$ is about $2 t_{c}$. This can be interpreted as follows. For large return periods, the maximum tends to approach the time of concentration (for reasons consistent with the rational method) as there is a large number of storms that may produce that flood, so the distribution of storm durations becomes relatively less important. The time of concentration (when the water from the furthest point of the catchment reaches the outlet) is, typically, larger than the response time (e.g. defined as the recession parameter), which may explain the maximum at twice the response time $\left(2 t_{c}\right)$.

It is clear that the distribution of possible storm durations is an important control on the mapping. Figure $6 \mathrm{a}$ and $\mathrm{b}$ are generalisations of Figs. 3 and 5 and show the effects of changing the mean storm duration. In Fig. 6a only the envelope curves are shown for $t_{c}=12 \mathrm{~h}$ while $\delta_{r}$ varies. The figure indicates that the average duration does not affect the position of this curve.

In Fig. $6 \mathrm{~b}$ the lines correspond to a return period of $T_{Q}=100$ years. The figure indicates that as $\delta_{r}$ increases, the maximum of the curves moves to the right, i.e., the ratio $t_{r}^{*} / t_{c}$ increases as well. This means that the critical storm duration depends both on catchment processes (here parameterised by $\left.t_{c}\right)$ and on rainfall processes $\left(\delta_{r}\right)$. It is interesting that the critical storm duration changes with the distribution of durations but the envelope curve $\max \left(T_{Q} / T_{P}\right)$ does not.

In Fig. $6 \mathrm{c}$ and d, the coefficient of variation $C V_{r}$ of the distribution of $t_{r}$ has been changed by varying the shape parameter $\beta_{r}$ of the Weibull distribution (see Eq. 4). As $C V_{r}$ tends to 0 , the flood return period approaches the rainfall return period (i.e., a 1 to 1 mapping) as only a single storm duration is possible. This is the case of Fig. 2a. On the other hand, if $C V_{r}$ increases, $\max \left(T_{Q} / T_{P}\right)$ decreases and reaches a stable value of about 0.3 for very dispersed distributions. This is because, for high $C V_{r}$, it is mainly the tail of the distribution that changes. For the durations commensurate with the catchment response time $t_{c}$, the changes are small.

\subsection{The catchment as a filter on storm durations}

In the previous sections we have derived flood and storm return periods for different storm durations. For example, considering Fig. $3 \mathrm{~b}$, a storm of duration $t_{r} \approx t_{c}$ would produce a flood of return period $T_{Q} \approx 10$ years if $T_{P} \approx 50$ years, provided such as storm occurs. Figure $3 \mathrm{a}$ and $\mathrm{b}$ gives no information about the probability of storm occurrence. It is now of interest to understand what is the probability that a maximum annual flood was produced by an event of duration $t_{r}$. The distribution of this probability is called here distribution of flood-producing storm durations, $f_{T_{r}}^{*}$. Some cases obtained by Monte-Carlo simulations are shown in Fig. 7. In both graphs, the Weibull parent distribution of $t_{r}$ of Eq. (2) is shown for comparison (solid line). The transition to the extremes transforms it into the dashed and dashed-dotted lines. As shown in Fig. 7a, the distribution of flood-producing storm durations depends on the catchment response time $t_{c}$ (here the exponential UH is used). Given a "parent" distribution of storm durations (e.g. Eq. 2), the catchment acts as a filter (see also Skøien and Blöschl, 2006). Storms with durations on the order of magnitude of the catchment response time are transformed into annual floods with higher probability. If $t_{c}$ increases, also the average and the spread of the distribution of flood-producing $t_{r}$ increase. In small catchments with short response times, it is usually the short duration storms that produce large floods (e.g. flash-floods), and the duration of the storms does not vary much. On the other hand, in large catchments with long response times, long duration storms tend to produce large floods and there is a larger variability of flood-producing storm durations. Short flash floods, however, rarely produce large floods in large catchments. Clearly, this is the conceptual basis of the rational method. 

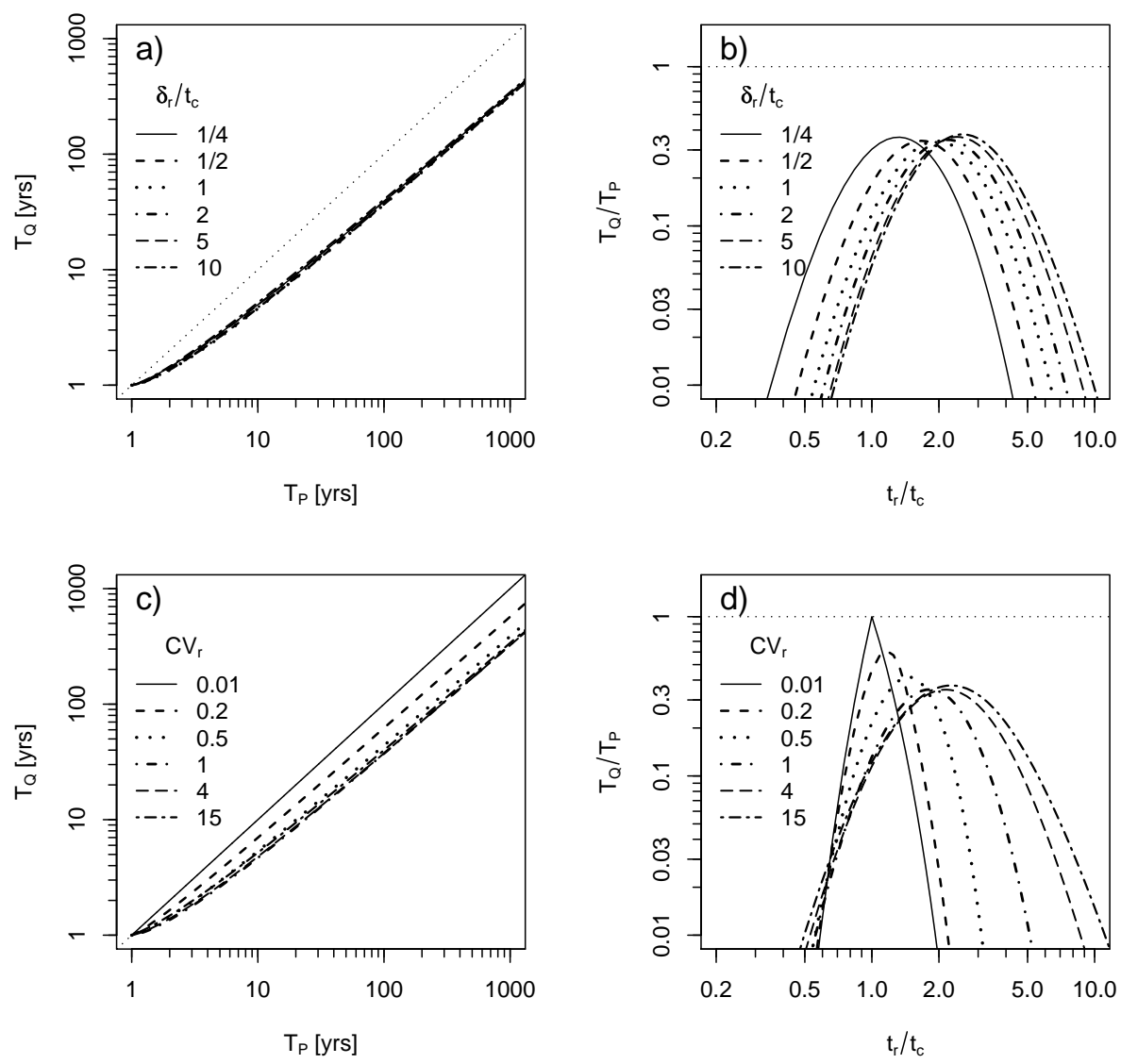

Fig. 6. Relation between rainfall return periods $T_{P}$ and flood return periods $T_{Q}$ for different distributions of the storm duration $t_{r}$ : (a, b) different relations between catchment response time $t_{c}$ and mean storm duration $\delta_{r}$ when the coefficient of variation $C V_{r} \approx 1.46\left(\beta_{r}=0.7\right)$ and $t_{c}=12 \mathrm{~h} ;(\mathbf{c}, \mathbf{d})$ different $C V_{r}$ when $\delta_{r}=t_{c}=12 \mathrm{~h} ;(\mathrm{a}, \mathrm{c})$ envelope curves, result of the design storm procedure; (b, d) horizontal slices for $T_{Q}=100$ years.
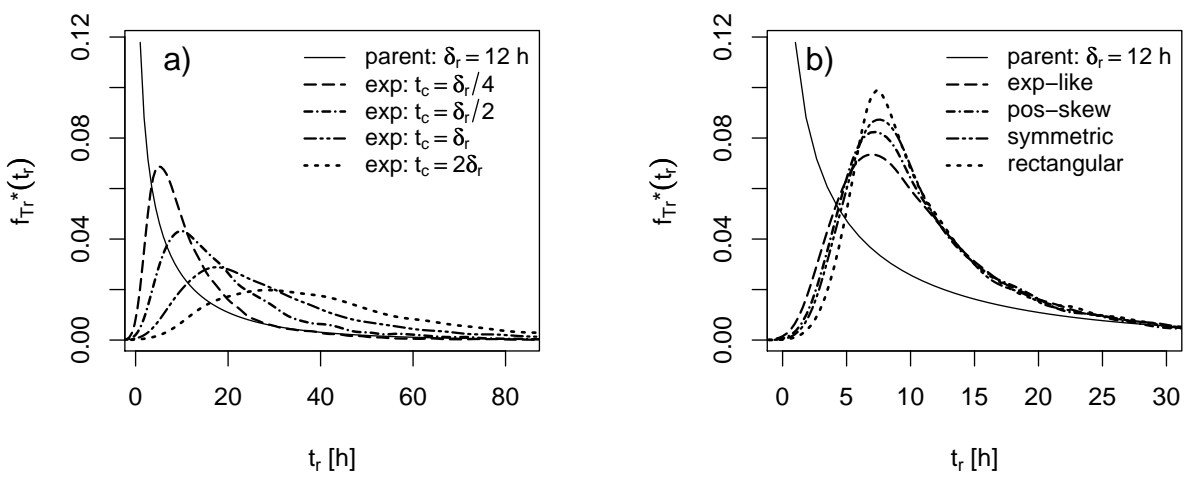

Fig. 7. Effect of the response time $t_{c}$ (a) and the shape of the UH (b) on the flood-producing storm durations. The black continuous line is the parent distribution, given in Eq. (2) with mean storm duration $\delta_{r}=12 \mathrm{~h}$ and coefficient of variation $C V_{r} \approx 1.46\left(\beta_{r}=0.7\right)$, while the other lines are the distributions of the durations of flood-producing storms. In panel (a) the UH is a linear reservoir (see Eq. 8) with response time $t_{c}=3,6,12,24 \mathrm{~h}$; in panel (b) the UHs have different shapes given by beta distributions with shape parameters $p_{\beta}$ and $q_{\beta}$ : exponential like ( $p_{\beta}=1, q_{\beta}=4$, width of $\left.17 \mathrm{~h}\right)$; positively skewed distribution $\left(p_{\beta}=2, q_{\beta}=4\right.$, width of $\left.12 \mathrm{~h}\right)$; symmetric distribution $\left(p_{\beta}=2, q_{\beta}=2\right.$, width of $9 \mathrm{~h})$; rectangular $\left(p_{\beta}=1, q_{\beta}=1\right.$, width of $\left.7 \mathrm{~h}\right)$. 
a)
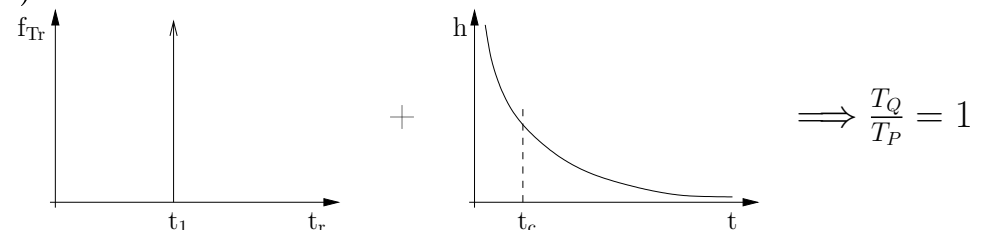

b)
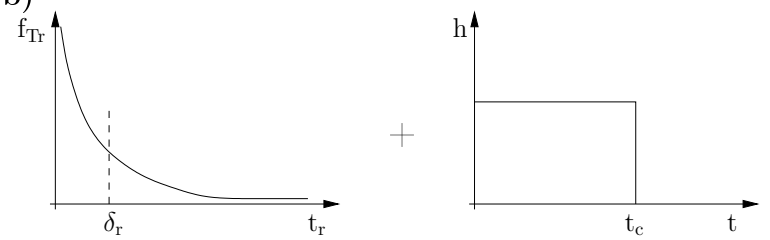

$\Longrightarrow\left\{\begin{array}{l}\frac{T_{Q}}{T_{P}}=1 \text { if } t_{r}=t_{c} \\ \frac{T_{Q}}{T_{P}}<1 \text { if } t_{r} \neq t_{c}\end{array}\right.$

c)
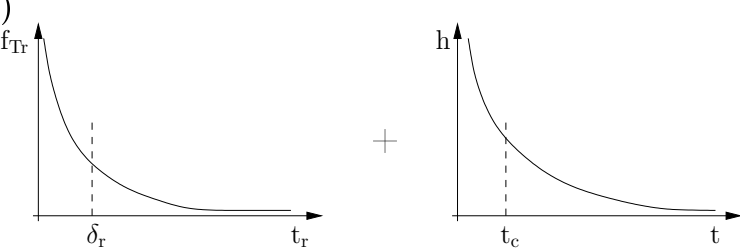

$\Longrightarrow \forall t_{r}, \frac{T_{Q}}{T_{P}}<1$

Fig. 8. Schematic summary of the results: (a) one possible storm duration $t_{1}$ and exponential UH; (b) Weibull distribution of the storm durations $t_{r}$ and rectangular $\mathrm{UH}$; (c) Weibull distribution of the storm durations $t_{r}$ and exponential $\mathrm{UH}$.

Figure $7 \mathrm{~b}$ shows instead the effects of the UH shape. We have considered five cases using a beta distribution for the UH with shape parameters $p_{\beta}$ and $q_{\beta}$ as in Alfieri et al. (2008). When $p_{\beta}=1$ and $q_{\beta}=4$, the $\mathrm{UH}$ is similar to the exponential one; when $p_{\beta}=2$ and $q_{\beta}=4$, it peaks at about $1 / 4$ of the UH width; when $p_{\beta}=2$ and $q_{\beta}=2$, it is symmetric with the maximum in the center; and when $p_{\beta}=1$ and $q_{\beta}=1$, it is a rectangular UH. The widths of the beta UHs have been chosen in order to have a similar mode of the distribution of flood-producing storm durations (about $7 \mathrm{~h}$ ), which implies similar response times.

The results show that the distribution of the durations of the flood-producing storms associated with the rectangular UH has the largest peak of all the distributions. This means that the rectangular filter is more selective on storm durations than the others. The exponential-like UH, in contrast, is the least selective. The difference in selectiveness of the exponential and rectangular filters is at the heart of the differences between the rainfall and flood return periods together with the fact that different rectangular filters are used for each $t_{\mathrm{IDF}}$ when evaluating the storm return periods.

\section{Conclusions}

The purpose of this research is to contribute to a better understanding of the design storm method used in engineering hydrology and a better understanding of the relationship between storm and flood return periods of observed floods. This relationship is controlled by many factors of which we examine storm duration as a starting point. Based on an an- alytical approach we derive the extreme storm and flood frequency distributions from a rainfall model assuming block rainfall, constant runoff coefficient and linear catchment response.

Even in the simple world modelled here, the relationship between storm and flood return periods is not straightforward. The main findings are summarised in Fig. 8:

a) If there is only a single storm duration, the flood return period $T_{Q}$ is always equal to the rainfall return period $T_{P}$, irrespective of the shape of the unit hydrograph.

b) In a more realistic case where storm durations vary, the flood and rainfall return periods are still identical, provided runoff response can be represented by a rectangular unit hydrograph and the storm duration is equal to the duration of the unit hydrograph. For longer and shorter storms $T_{Q}<T_{P}$.

c) In the more general case of a non-rectangular right skewed UH and varying storm durations, $T_{Q}<T_{P}$ always. This is because of the difference in the selectiveness of the rectangular filter used to construct the IDF curve and the UH, together with the fact that different rectangular filters are used for each $t_{\mathrm{IDF}}$ when evaluating the storm return periods.

A critical storm duration $t_{r}^{*}$ exists where $T_{Q} / T_{P}$ is at a maximum. For case (c), $t_{r}^{*}$ is (in decreasing importance) a function of the catchment response time $t_{c}$, average storm duration $\delta_{r}$, the coefficient of variation of the storm duration $C V_{r}$ and the rainfall return period $T_{P} . t_{r}^{*}$ ranges between $t_{c}$ 
and $3 t_{c}$, mainly depending on $\delta_{r}$. The maximum of $T_{Q} / T_{P}$ (when varying storm duration) is about 0.4 implying that, for the simple world examined here, the design storm method would give a 40 year flood when using a 100 year storm as an input to the runoff model.

We also examined the duration of the storms that produce the maximum annual floods. As the catchment response time $t_{c}$ increases, so does the average and the spread of the distribution of flood-producing storm durations. This analysis also confirmed the higher selectiveness of the rectangular filters used to calculate the IDF curves as compared to the exponential unit hydrograph.

Comprehensive sensitivity analyses (not shown in this paper) indicate that the above results are generic and do not depend much on the particular rainfall model used. For a world where

- storm duration varies,

- rainfall intensities are distributed according to a positively skewed distribution,

- extreme rainfall intensity decreases with storm duration and considering the simplifying assumptions made in this paper

- block rainfall

- constant runoff coefficient

- linear catchment response

the mapping of rainfall to flood return periods will always look very similar to the results shown here.

While these results are only applicable to this simple world, they are an important stepping stone towards the more complex cases involving random runoff coefficients and complex hyetographs, for example. For these, one would expect that the envelope curve in the $\left(T_{P}, T_{Q}\right)$ space goes above the 1 to 1 line as is observed for floods that have occurred (e.g. Gutknecht et al., 2002). These additional factors will be examined in a follow up paper.

\section{Notation}

Variables:

$T_{Q}$ flood return period [yrs]

$T_{P}$ storm return period [yrs]

$T_{\text {IDF }}$ IDF return period [yrs]

$t_{r}, t_{1}, t_{2}$ storm duration [h]

$i$ average storm intensity $[\mathrm{mm} / \mathrm{h}]$ over its duration $t_{r}$

$t_{\text {IDF }}$ aggregation level [h]

$\phi$ average storm intensity $[\mathrm{mm} / \mathrm{h}]$ over the aggregation level $t_{\mathrm{IDF}}$ $q_{p}$ flood peak $[\mathrm{mm} / \mathrm{h}]$

$t_{r}^{*}$ critical storm duration $[\mathrm{h}]$

$R, R^{\prime}$ regions of the $\left(i, t_{r}\right)$ space

$Y, Q, I, P$ random variables

Parameters (values for the basic case are indicated):

$\delta_{r}$ mean storm duration (12 h)

$C V_{r}$ coefficient of variation of the storm duration (1.46)

$\beta_{r}$ Weibull shape parameter of storm duration (0.7)

$\gamma_{r}$ Weibull scale parameter of storm duration $(9.48 \mathrm{~h})$

$\lambda$ Gamma scale parameter of storm intensity

$\kappa$ Gamma shape parameter of storm intensity

$a_{1}, b_{1}, a_{2}, b_{2}$ rainfall model parameters $\left(a_{1}=1.05 \mathrm{~mm} \mathrm{~h}^{-b_{1}-1}\right.$, $b_{1}=0.01, a_{2}=1.5, b_{2}=-0.55$ )

$m$ average number of storms per year (40)

$p_{\beta}, q_{\beta}$ shape parameters of the Beta distribution

Operators:

$E($.$) expected value$

$C V$ (.) coefficient of variation

$F_{X}($.) cumulative distribution function of $X$

$f_{X}($.$) probability density function of X$

$p_{X}($.$) probability mass function of X$

$f_{X, Y}($.$) joint probability density function of X$ and $Y$

$f_{X \mid Y}($.$) conditional probability density function of X$ given $Y$

$\operatorname{Pr}($.$) probability$

$\Gamma$ (.) Gamma function

$\Pi_{Q}($.) runoff model operator

$\Pi_{P}($.$) IDF filter operator$

Acknowledgements. We gratefully acknowledge financial support from the HYDRATE (Hydrometeorological data resources and technologies for effective flash flood forecasting) project funded by the European Union and the Mountain flood project funded by the Austrian Academy of Sciences as part of their ISDR programme. We would like to thank Lars Gottschalk for useful comments on an early draft of the manuscript and Jürgen Komma for precious help with the Monte-Carlo simulations. We would also like to thank Attilio Castellarin, Jon Olav Skøien and an anonymous reviewer for their useful comments on the manuscript in HESSD.

Edited by: F. Pappenberger 


\section{References}

Alfieri, L., Laio, F., and Claps, P.: A simulation experiment for optimal design hyetograph selection, Hydrol. Process., 22(6), 813820, doi:10.1002/hyp.6646, 2008.

Bradley, A. A. and Potter, K. W.: Flood frequency analysis of simulated flows, Water Resour. Res., 28, 2375-2385, 1992.

Chow, V. T., Maidment, D. R., and Mays, L. W.: Applied Hydrology, Civil Engineering Series, McGraw-Hill Book Company, International Edn., 572 pp., 1988.

Gutknecht, D., Reszler, C., and Blöschl, G.: Das Katastrophenhochwasser vom 7. August 2002 am Kamp - eine erste Einschätzung (The August 7, 2002 - flood of the Kamp - a first assessment), Elektrotechnik und Informationstechnik, 119, 411413, 2002.

Kottegoda, N. T. and Rosso, R.: Statistics, Probability, and Reliability for Civil and Environmental Engineers, McGraw-Hill Companies, International Edn., 735 pp., 1997.

Linsley, R. K., Kohler, M. A., and Paulhus, J. L. H.: Hydrology for Engineers, McGraw-Hill Book Company, London, Si Metric Edn., 492 pp., 1988.
Packman, J. C. and Kidd, C. H. R.: A logical approach to the design storm concept, Water Resour. Res., 16, 994-1000, 1980.

Pilgrim, D. H. and Cordery, I.: Rainfall temporal patterns for design floods, J. Hydr. Eng. Div.-ASCE, 101, 81-95, 1975.

Pilgrim, D. H. and Cordery, I.: Flood Runoff, in: HandBook of Hydrology, edited by Maidment, D. R., McGraw-Hill Companies, International Edn., Chap. 9, 42 pp., 1993.

Sivapalan, M. and Blöschl, G.: Transformation of point rainfall to areal rainfall: Intensity-duration frequency curves, J. Hydrol., 204, 150-167, 1998.

Sivapalan, M., Blöschl, G., Merz, R., and Gutknecht, D.: Linking flood frequency to long-term water balance: Incorporating effects of seasonality, Water Resour. Res., 41, W06012, doi: 10.1029/2004WR003439, 2005.

Skøien, J. O. and Blöschl, G.: Catchments as space-time filters - a joint spatio-temporal geostatistical analysis of runoff and precipitation, Hydrol. Earth Syst. Sci., 10, 645-662, 2006, http://www.hydrol-earth-syst-sci.net/10/645/2006/.

Wood, E. F.: An analysis of the effects of parameter uncertainty in deterministic hydrologic models, Water Resour. Res., 12, 925932, 1976. 